CUBO A Mathematical Journal

Vol.12, $N^{\underline{O}}$ 01, (219-230). March 2010

\title{
Strong Vector Equilibrium Problems in Topological Vector Spaces Via KKM Maps
}

\author{
A.P. FARAJZADEH \\ Department of Mathematics, Razi University, \\ Kermanshah, 67149, Iran \\ email: ali-ff@sci.razi.ac.ir \\ A. Amini-Harandi* \\ Department of Mathematics, University of Shahrekord, \\ Shahrekord, 88186-34141, Iran \\ email: aminih_a@yahoo.com \\ D. O'REGAN \\ Department of Mathematics, National University of Ireland, \\ Galway, Ireland \\ email: donal.oregan@nuigalway.ie \\ AND \\ R.P. Agarwal \\ Department of Mathematical Sciences, Florida Institute of Technology, \\ Melbourne, FL 32901, USA \\ email: agarwal@fit.edu
}

\begin{abstract}
In this paper, we establish some existence results for strong vector equilibrium problems (for short, SVEP) in topological vector spaces. The solvability of the SVEP is presented using the
\end{abstract}

*The second author was in part supported by a grant from IPM (No. 85470015) 
Fan-KKM lemma. These results give a positive answer to an open problem proposed by Chen and Hou and generalize many important results in the recent literature.

\section{RESUMEN}

En este artículo, establecemos algunos resultados de existencia para problemas de equilibrio strong vector en espacios vectoriales topológicos (abreviadamente, SVEP). La salubilidad del SVEP es presentada usando el lema de Fan-KKM. Estos resultados dan una respuesta positiva a problemas abiertos propuestos por Chen y Hon y generalizan varios resultados importantes en la literatura reciente.

Key words and phrases: Strong vector equilibrium, Upper sign continuity, Pseudomonotone bifunction, Quasimonotone bifunction.

Math. Subj. Class.: PLEASE INFORM

\section{Introduction}

It is well known that the vector equilibrium problem provides a unified model for several classes of problems, for example, vector variational inequality problems, vector complementarity problems, vector optimization problems, and vector saddle point problems ( see $[1,8]$ ). Let $X$ and $Y$ be two real Hausdorff topological vector spaces, and $K$ a nonempty subset of $X$. Let $F: K \times K \rightarrow Y$ be a given bifunction and $W: K \rightarrow 2^{Y}$ be a set valued mapping. Let $C \subseteq Y$. $C$ is said a convex cone subset of $Y$ if, $\lambda C \subseteq C$ for each $\lambda \geq 0$ and $C+C \subseteq C$. The convex cone $C$ is called pointed if, $C \cap(-C)=\{0\}$. In this paper, we consider the following problem :

Strong vector equilibrium problem (for short, SVEP) which consists in finding $\bar{x} \in K$ such that

$$
F(\bar{x}, y) \in W(\bar{x}), \quad \forall y \in K
$$

If $X$ and $Y$ be two Banach spaces and $F(x, y)=\langle T(x), y-x\rangle$, where $T: K \rightarrow L(X, Y)$ and $L(X, Y)$ denotes the set of all continuous linear mappings from $X$ into $Y$, then SVEP reduces to the strong vector variational inequality SVVI which was considered by Fang and Huang [7]. In [7] Fang and Huang obtained some existence results for SVVI which gave a positive answer to the open problem proposed by Chen and Hou [3] in a real Banach space. The main purpose of this work is to establish some existence results for SVEP in a real Hausdorff topological vector spaces. Our results extend and improve the corresponding results of Fang and Huang [6], Iusem and Sosa [9] and many others.

The paper is organized as follows. In the rest of this section we recall some notation,definitions, and the Fan-KKM lemma which are used in the next section. In section 2, we first present the solvability of SVEP without monotonicity by using the Fan-KKM lemma and then obtain an existence result for SVEP with generalized monotonicity. 
Throughout the paper, unless otherwise specified, let $X$ and $Y$ be two real Hausdorff topological vector spaces. For given bifunction $F: K \times K \rightarrow Y$ and $f \in Y^{*}=L(Y, \mathbb{R})$ consider $f \circ F: K \times K \rightarrow \mathbb{R}$ as $\langle f \circ F,(x, y)\rangle=f(\langle F,(x, y)\rangle)$. We denote the duality pairing between $X^{*}$ and $X$, by $\langle.,$.$\rangle , and the open line segment joining between x, y \in K$ by $] x, y[$. Let $A$ be a nonempty subset of a topological space $X$. We denote by $2^{A}$ the family of all subsets of the set $A$ and by $\mathcal{F}(A)$ the family of all nonempty finite subsets of $A$. In a topological vector space $X$, let $i n t, c l$, and co denote the interior, closure and convex hull respectively.

Let $K$ be a nonempty convex subset of $X$ and let $K_{0}$ be a subset of $K$. A multivalued map $\Gamma: K_{0} \rightarrow 2^{K}$ is said to be a KKM map if

$$
c o A \subseteq \bigcup_{x \in A} \Gamma(x), \quad \forall A \in \mathcal{F}\left(K_{0}\right) .
$$

Let $X$ and $Y$ be two topological spaces. The set-valued mapping $T: X \rightarrow 2^{Y}$ is called upper semi-continuous (u.s.c.) at $x \in X$ if for each open set $V$ containing $T(x)$ there is an open set $U$ containing $x$ such that for each $t \in U, T(t) \subseteq V$; $T$ is said to be u.s.c. on $X$ if it is u.s.c. at all $x \in X$. The map $T$ is said to be closed if the set $\operatorname{Gr}(T)=\{(x, y): x \in X, y \in T(x)\}$ is a closed set in $X \times Y$.

Proposition 1.1. If $T$ is closed and $\overline{T(X)}=\overline{\bigcup_{x \in K} T(x)}$ is compact, then $T$ is u.s.c.

We need the following theorems which are special cases of [5, Theorem 3.1] and [12, Theorem 2.1 ], respectively, in the sequel.

Theorem 1.2. ([5]). Let $K$ be a nonempty subset of a topological vector space $X$ and $F: K \rightarrow 2^{X}$ be a KKM mapping with closed values. Assume that there exist a nonempty compact convex subset $B$ of $K$ such that $D=\bigcap_{x \in B} F(x)$ is compact. Then

$$
\bigcap_{x \in K} F(x) \neq \emptyset
$$

\section{The Main Results}

The following theorem provides sufficient conditions in order to guarantee nonemptyness and compactness of the solution set of SVEP.

Theorem 2.1. Let $X$ and $Y$ be real Hausdorff topological vector spaces, $K$ a nonempty closed subset of $X, F: K \times K \rightarrow Y$ and $W: K \rightarrow 2^{Y}$ be two set valued mappings. Assume the following hypotheses hold

(a) for all finite subsets $A$ of $K$ and for all $x \in$ coA there exists $y \in A$ such that $F(x, y) \in W(x)$; 
(b) for each $y \in K$, the set $\{x \in K: F(x, y) \in W(x)\}$ is closed

(c) there exist a nonempty compact subset $B$ of $K$ and a nonempty convex compact subset $D$ of $K$ such that, for each $x \in K \backslash B$ there exists $y \in D$ such that $F(x, y) \notin W(x)$.

then, the solution set of SVEP is nonempty and compact.

Proof. We define $\Gamma: K \rightarrow 2^{K}$ as follows

$$
\Gamma(y)=\{x \in K: F(x, y) \in W(x)\} .
$$

By (a),$\Gamma$ is a KKM mapping. Applying (b) and (c), we deduce that $\bigcap_{y \in D} \Gamma(y)$ is a closed subset of $B$. Now, $\Gamma$ satisfies all of the assumptions of Theorem 1.2 and hence $\bigcap_{x \in K} \Gamma(x) \neq \emptyset$. This means that SVEP has a solution. By (b), the solution set of SVEP is closed and by (c) it is subset of the compact set $B$. Thus the solution set of SVEP is compact and the proof is complete.

Remark 2.2. $\quad$ (i) If $K$ is convex, $F(x, x) \in W(x), \quad C(x)=Y \backslash W(x)$ is a convex cone, for all $x \in K$, and the mapping $F$ is concave in $y$ with respect to $C(x)$, that is,

$$
F\left(x, t y_{1}+(1-t) y_{2}\right)-\left(t F\left(x, y_{1}\right)+(1-t) F\left(x, y_{2}\right)\right) \in C(x),
$$

for each $x, y_{1}, y_{2} \in K$ and $\left.t \in\right] 0,1[$ then condition (a) of Theorem 2.1 holds. To see this let $A=\left\{y_{1}, \ldots, y_{n}\right\}$ be a finite subset of $K$ and $x=\sum_{i=1}^{n} \lambda_{i} y_{i} \in \operatorname{co}(A)$ where $\lambda_{i} \geq 0$ and $\sum_{i=1}^{n} \lambda_{i}=1$. On the contrary, we suppose that (a) does not hold. Then for each $1 \leq i \leq n$, $F\left(x, y_{i}\right) \notin W(x)$. Thus

$$
F\left(x, y_{i}\right) \in C(x), \quad \text { for each } \quad 1 \leq i \leq n .
$$

Since $F$ is concave in $y$ with respect to $C(x)$ we get

$$
F\left(x, \Sigma_{i=1}^{n} \lambda_{i} y_{i}\right)-\sum_{i=1}^{n} \lambda_{i} F\left(x, y_{i}\right) \in C(x) .
$$

By $(2.1)$, note $C(x)$ is convex, we have

$$
\sum_{i=1}^{n} \lambda_{i} F\left(x, y_{i}\right) \in C(x)
$$

Now since $C(x)$ is a convex cone, by $(2.2)$ and (2.3) we obtain

$$
F(x, x)=F\left(x, \Sigma_{i=1}^{n} \lambda_{i} y_{i}\right) \in C(x),
$$

which contradicts our assumption. For example if we let $X=Y=\mathbb{R}, W(x)=(-\infty, 0]$, and define $F(x, y)=x-y$ then $F$ satisfies condition (a).

(ii) It is clear that condition (a) is different from diagonally quasiconvex, in the single valued case, defined in [4, page 114]. 
(iii) If the mapping $F$ is continuous in $x$, for all fixed $y \in K$ and the graph of the mapping $W$ is closed then $D=\{x \in K: F(x, y) \in W(x)\}$ is closed and so condition (b) holds. To see this Let $x_{n} \in D$ and $x_{n} \rightarrow x \in K$. Then for each $n \in \mathbb{N}, F\left(x_{n}, y\right) \in W\left(x_{n}\right)$. Since $F(., y)$ is continuous and the graph of $W$ is closed then $F(x, y) \in W(x)$ which shows that $x \in D$. Finally if $K$ is compact then condition (c) trivially holds.

In the following, as an application of Theorem 2.1, we give a topological vector space version of Theorem 2.1 of Fang and Huang [7] for a family of moving closed pointed convex cones $\{C(x)$ : $x \in K\}$.

Corollary 2.3. Let $K$ be a nonempty closed convex subset of $X$ and $T: K \rightarrow L(X, Y)$ be a mapping such that

$\left(a^{\prime}\right)$ for each $y \in K$, the set $\{x \in K:\langle T x, y-x\rangle \in-C(x) \backslash\{0\}\}$ is open in $K$,

$\left(b^{\prime}\right)$ there exist a nonempty compact subset $B$ of $K$ and a nonempty convex compact subset $D$ of $K$ such that, for each $x \in K \backslash B$ there exists $y \in D$ such that $\langle T x, y-x\rangle \in-C(x) \backslash\{0\}$.

Then the set $\{x \in K:\langle T x, y-x\rangle \notin-C(x) \backslash\{0\}, \forall y \in K\}$ is a nonempty and compact subset of $K$.

Proof. Let $F(x, y)=\langle T(x), y-x\rangle$ and $W(x)=Y \backslash(-C(x) \backslash\{0\})$. We claim that $F$ and $W$ satisfy all of the assumptions of Theorem 2.1. Indeed, by $\left(a^{\prime}\right)$, the set $\{x \in K: F(x, y) \in W(x)\}$ is closed in $K$ (so closed in $X$ ), for all $y \in K$. Now if for a finite subset $A=\left\{y_{1}, y_{2}, \ldots, y_{n}\right\}$ of $K$ there exists $x \in \operatorname{co} A$ such that $F(x, y) \notin W(x)$, for all $y \in A$. Then

$$
F\left(x, y_{i}\right) \in-C(x) \backslash\{0\}, \quad \text { for each1 } \leq i \leq n .
$$

Since $C(x)$ is closed pointed convex cone then $-C(x) \backslash\{0\}$ is a convex cone and so from the definition of $F$ we get (here $x=\sum_{i=1}^{n} \lambda_{i} y_{i}$ with $\lambda_{i} \geq 0$ and $\sum_{i=1}^{n} \lambda_{i}=1$ )

$$
0=F(x, x)=F\left(x, \sum_{i=1}^{n} \lambda_{i} y_{i}\right)=\sum_{i=1}^{n} \lambda_{i}\left\langle T x, y_{i}-x\right\rangle \in-C(x) \backslash\{0\},
$$

which is a contradiction. This proves (a) of Theorem 2.1. Finally $\left(b^{\prime}\right)$ guarantees condition (c) of Theorem 2.1 and so the proof is complete.

In what follows we give another application of Theorem 2.1 in order to provide sufficient conditions that a function defined on a nonempty convex subset of a normed space has a fixed point.

Corollary 2.4. Let $K$ be a nonempty, closed convex subset of a real normed vector space $X$ and let $f: K \rightarrow K$ be a continuous mapping. If there is a nonempty compact subset $B$ of $K$ and a compact convex subset $D$ of $K$ such that for every $x \in K$ there exists $y \in D$ satisfying $\|x-f(x)\|>\|y-f(x)\|$, then there exists $\bar{x} \in K$ such that $\bar{x}=f(\bar{x})$. Furthermore, the set of all such elements is a compact subset of $B$. 
Proof. Define $F: K \times K \rightarrow \mathbb{R}$ as

$$
F(x, y)=-\|y-f(x)\|+\|x-f(x)\|, \quad \forall x, y \in K,
$$

and let $W(x)=[-\infty, 0) \subset Y=\mathbb{R}$, for all $x \in K$. The function $F$ is concave in the second variable with respect to the set $(Y \backslash W(x))=[0, \infty)$ which is a convex subset of $Y$. Hence by Remark 2.2 (i), $F$ satisfies condition (a) of Theorem 2.1. By the continuity of $F$ in the first variable and the closedness of graph of $W$ (see Remark 2.2 (iii)) we deduce that $F$ satisfies condition (b) of Theorem 2.1. Finally $F$ satisfies condition (c) of Theorem 2.1 by assumption. Thus there exists $\bar{x} \in K$ such that

$$
F(\bar{x}, y)=-\|y-f(\bar{x})\|+\|\bar{x}-f(\bar{x})\| \leq 0, \text { for all } y \in K .
$$

Now, if, in the previous relation, we take $y=f(\bar{x})$ then $\|\bar{x}-f(\bar{x})\| \leq 0$ which implies the result requested.

Remark 2.5. Corollary 2.4 generalizes Theorem 3.2 in [12] for infinite dimensional normed spaces.

Now using a scalarization method, we establish an existence theorem for SVEP. In particular, we prove the following theorem which is an extension of Theorem 2.2 in [7].

Theorem 2.6. Let $K$ be a nonempty, closed convex subset of a real Hausdorff topological vector space $X, W$ be a nonempty subset of $X$. Let $F: K \times K \rightarrow Y$ be a bifunction. Suppose that there exists $x_{0} \in K$ and $f \in\left\{y^{*} \in Y^{*}:\left\langle y^{*}, c\right\rangle<0, \quad \forall c \in Y \backslash W\right\}$ such that

(a) $F(x, x)=0$, for all $x \in K$;

(b) for each $x, y, z \in K$, if $f \circ F(x, y) \leq 0$ and $f o F(x, z)<0$, then $f o F(x, u)<0, \forall u \in] y, z[$;

(c) for each $y \in K$, the set $\{x \in K: f o F(x, y) \geq 0\}$ is closed in $K$;

(d) there exist a nonempty compact subset $B$ of $K$ such that,

$$
\forall x \in K \backslash B, \quad f o F\left(x, x_{0}\right)<0 .
$$

Then there exists $\bar{x} \in K$ such that $F(\bar{x}, y) \in W$, for all $y \in K$.

Proof. Define $G: K \rightarrow 2^{K}$ by $G(y)=\{x \in K: f o F(x, y) \geq 0\}$. By (c), $G(y)$ is closed for each $y \in K$. Now we show that $G$ is a KKM map. Let $A=\left\{y_{1}, y_{2}, \ldots, y_{n}\right\}$ be a finite subset of $K$ and $x=\sum_{i=1}^{n} \lambda_{i} y_{i} \in \operatorname{co}(A)$ where $\lambda_{i} \geq 0$ and $\sum_{i=1}^{n} \lambda_{i}=1$. We prove that $x \in \cup_{i=1}^{n} G\left(y_{i}\right)$. On the contrary, suppose for each $i=1, \ldots, n, x \notin G\left(y_{i}\right)$. Consequently

$$
f \circ F\left(x, y_{i}\right)<0, \quad \text { for each } i=1, \ldots, n \text {. }
$$


Notice that using induction, we can deduce from (b) that for each $x, y_{1}, \ldots, y_{n} \in K$, if for each $i=1, \ldots, n, f \circ F\left(x, y_{i}\right)<0$ then $f \circ F(x, u)<0$ for each $u \in c o\left(y_{1}, y_{2}, \ldots, y_{n}\right)$. Thus by (2.4) we get

$$
0=f \circ F(x, x)<0,
$$

which is a contradiction. Let $D=\left\{x_{0}\right\}$, then $\cap_{y \in D} G(y)=G\left(x_{0}\right) \subseteq B$ by (d). Thus Theorem 1.2. implies $\bigcap_{y \in K} G(y) \neq \emptyset$. Let $\bar{x} \in \bigcap_{y \in K} G(y)$, then

$$
f \circ F(\bar{x}, y) \geq 0, \quad \text { for all } \quad y \in K \text {. }
$$

Now, if $F(\bar{x}, y) \notin W$, for some $y \in K$, then from $f \in\left\{y^{*} \in Y^{*}:\left\langle y^{*}, c\right\rangle<0, \quad \forall c \in Y \backslash W\right\}$, we get $f o F(\bar{x}, y)<0$, which is a contradiction by $(2.5)$.

As an application of Theorem 2.6 we now present two corollaries. The first is a topological version of Theorem 2.2 in [7] with mild assumptions and the second improves Theorem 3.12 in [10].

Corollary 2.7. Let $K$ be a nonempty closed convex subset of a topological vector space $X, C$ be a nonempty closed pointed convex cone of $X$ and let $T: K \rightarrow L(X, Y)$ be a nonlinear mapping. Suppose that there exist $x_{0} \in K, f \in\left\{y^{*} \in Y^{*}:\left\langle y^{*}, c\right\rangle>0, \forall c \in C \backslash\{0\}\right\}$, and a nonempty compact subset $B$ of $K$ such that

$$
\forall x \in K \backslash B, \quad f o T(x)\left(x-x_{0}\right)<0 .
$$

Then there exists $\bar{x} \in K$ such that $\langle T \bar{x}, y-\bar{x}\rangle \notin-C \backslash\{0\}$, for all $y \in K$.

Proof. Define $F: K \times K \rightarrow Y$ by $F(x, y)=\langle T x, y-x\rangle$ and $W=Y \backslash-C$. Now the result follows of Theorem 2.6.

Corollary 2.8. Suppose that $(X,\|\|$.$) is a real reflexive Banach space and K$ is a nonempty closed convex subset of $X$. Let $F: K \times K \rightarrow \mathbb{R}$ be a bifunction such that:

(a) $F(x, x)=0, \quad$ for each $x \in K$,

(b) for each $x, y, z \in K$, if $F(x, y) \geq 0$ and $F(x, z)>0$, then $F(x, u)>0, \forall u \in] y, z[$;

(c) for each $y \in K$, the set $\{x \in K: F(x, y) \geq 0\}$ is closed;

(d) there exists $r_{0}>0$ such that for each $x \in K \backslash K_{r_{0}}$ there exists $y \in K_{r_{0}}$ with $F(x, y) \leq 0$, where $K_{r_{0}}=\left\{x \in K:\|x\| \leq r_{0}\right\}$.

Then, the solution set of the equilibrium problem,i.e., $\quad\{x \in K: F(x, y) \leq 0, \quad \forall y \in K\}$, is nonempty and compact. 
Proof. Set $Y=\mathbb{R}, W(x)=[0, \infty)$ and define $f: \mathbb{R} \rightarrow \mathbb{R}$ by $f(x)=x$ for each $x \in K$. By (b), note $f \circ F=F$, so we get condition (b) of Theorem 2.6. Pick $B=D=K_{r_{0}}$, since $K_{r_{0}}$ is weakly compact convex, then condition (d) of the Theorem 2.6 trivially holds and so Theorem 2.6 implies that there is $\bar{x} \in K$ such that $F(\bar{x}, y) \geq 0$, for all $y \in K$. Moreover, by (b) and (d), the set $\{x \in K: F(x, y) \geq 0, \quad \forall x \in K\}$, is a closed subset of $K_{r_{0}}$, respectively. This completes the proof.

Definition 2.9. Let $F: K \times K \rightarrow Y$, be a given bifunction and $\mathcal{C}=\{C(x): x \in K\}$ is a family of closed convex cone proper subsets of $Y$. We say $F$ is $\mathcal{C}$-psudomonotone if the following implication holds

$$
F(x, y) \notin-C(x) \backslash\{0\} \Rightarrow F(y, x) \in-C(y) .
$$

A nonlinear mapping $T: K \rightarrow L(X, Y)$ is $C$-psudomonotone if the bifunction $F(x, y)=\langle T x, y-x\rangle$ is $C$-psudomonotone.

Remark 2.10. For the single-valued bifunction $F$, our definition of pseudomonotonicity reduces to that in [11]. Moreover, if we let $F(x, y)=\langle T(x), y-x\rangle$, where $T: K \rightarrow L(X, Y)$ is a nonlinear mapping, and $C(x)=C$ for all $x \in K, \quad C$ is a convex cone of $Y$, then the previous definition reduces to Definition 2.1 in [7].

Definition 2.11. Let $F: K \times K \rightarrow Y$, and $\mathcal{C}=\{C(x): x \in K\}$ is a family of convex cone proper subsets of $Y$. We say that $F$ is $\mathcal{C}$-upper sign continuous if the following implication holds for every $x, y \in K$,

$$
F(u, y) \notin-C(u) \backslash\{0\}, \quad \forall u \in] x, y[\Rightarrow F(x, y) \notin-C(x) \backslash\{0\} .
$$

A nonlinear mapping $T: K \rightarrow L(X, Y)$ is $\mathcal{C}$-upper sign continuous if the bifunction $F(x, y)=$ $\langle T x, y-x\rangle$ is $\mathcal{C}$-upper sign continuous.

Remark 2.12. If $Y=\mathbb{R}$ and $C(x)=[0, \infty)$, for all $x \in \mathbb{R}$, then our definition of upper sign continuity, reduces to the definition of upper sign continuity introduced by Bianchi and Pini in [2]. Also, if the graph of the set-valued map $W: K \rightarrow 2^{Y}$ defined by $W(x)=Y \backslash-C(x) \backslash\{0\}$ is closed and the function $t \rightarrow F\left(x_{t}, y\right)$ is continuous at $t=0$, where $x_{t}=(1-t) x+t y$, then $F$ is $\mathcal{C}$ - upper sign continuous. This shows that if we set $F(x, y)=\langle T(x), y-x\rangle$, where $T$ is nonlinear mapping, and $C(x)=C$ for all $x \in K, \quad C$ is a closed convex cone of $Y$, then hemicontinuity of $T$ (Definition 2.2 in [6]) implies $\mathcal{C}$-upper sign continuity of $F$.

Lemma 2.13. Let $K$ be a nonempty closed convex subset of $X$, and $\mathcal{C}=\{C(x): x \in K\}$ is a family of convex cone proper subsets of $Y$. Let $F: K \times K \rightarrow Y$ be a $\mathcal{C}$-pseudomonotone and $\mathcal{C}$-upper sign continuous function. Assume that the following assumptions hold,

(a) $F(x, x)=0$, for each $x \in K$,

(b) for each $x, y, z \in K$, if $F(x, y) \in-C(x) \backslash\{0\}$ and $F(x, z) \in-C(x)$, then $F(x, u) \in-C(x) \backslash\{0\}$, for all $u \in] y, z[$, 
then for every $x_{0} \in K$,

$$
F\left(x_{0}, y\right) \notin-C\left(x_{0}\right) \backslash\{0\}, \quad \forall y \in K
$$

if and only if

$$
F\left(y, x_{0}\right) \in-C(y), \quad \forall y \in K .
$$

Proof. Let $x_{0} \in K$ be such that

$$
F\left(x_{0}, y\right) \notin-C\left(x_{0}\right) \backslash\{0\}, \quad \forall y \in K .
$$

Now the $\mathcal{C}$-Pseudomonotonicity of $F$, implies that

$$
F\left(y, x_{0}\right) \in-C(y), \quad \forall y \in K
$$

Conversely, suppose that

$$
F\left(y, x_{0}\right) \in-C(y), \quad \forall y \in K .
$$

We first show that for each $y \in K$,

$$
u \in] x_{0}, y[\Rightarrow F(u, y) \notin-C(u) \backslash\{0\} .
$$

On the contrary, we suppose that there exist $y \in K$ and $u \in] x_{0}, y[$ such that

$$
F(u, y) \in-C(u) \backslash\{0\} .
$$

By our assumption,

$$
F\left(u, x_{0}\right) \in-C(u) .
$$

From (2.7), (2.8) and (b) we get

$$
0=F(u, u) \in-C(u) \backslash\{0\},
$$

which is a contradiction. Thus, $(2.6)$ holds. Since $F$ is $\mathcal{C}$-upper sign continuous, (2.6) implies that

$$
F\left(x_{0}, y\right) \notin-C\left(x_{0}\right) \backslash\{0\} .
$$

The following result improves Proposition 2.5 in [10].

Corollary 2.14. Let $K$ be a nonempty convex subset of $X$ and $F: K \times K \rightarrow \mathbb{R}$ be a pseudomonotone bifunction satisfying the following conditions: 
(a) for each $x \in K, \quad F(x, x)=0$,

(b) for each $x, y, z \in K$, if $F(x, y)<0$ and $F(x, z) \leq 0$, then $F(x, u)<0$, for all $u \in] y, z[$,

(c) for every $x$ and $y$ in $K$ the following implication holds:

$$
F(u, y) \geq 0, \forall u \in] x, y[\Rightarrow F(x, y) \geq 0 .
$$

Let $x_{0} \in K$, and then

$$
F\left(x_{0}, y\right) \geq 0, \quad \forall y \in K \Leftrightarrow F\left(y, x_{0}\right) \leq 0, \quad \forall y \in K
$$

Proof. In the previous Lemma, let $Y=\mathbb{R}$ and $C(x)=[0, \infty)$, for every $x \in K$. Obviously, $F$ is $\mathcal{C}-$ pseudomonotone by (b) and $\mathcal{C}$-upper sign continuous by (c). Now, the result follows from Lemma 2.13 .

Theorem 2.15. Let $K$ be a nonempty convex subset of $X$ and $\mathcal{C}=\{C(x): x \in K\}$ is a family of convex cone proper subsets of $Y$. Let $F: K \times K \rightarrow Y$ be a $\mathcal{C}$-pseudomonotone and $\mathcal{C}$-upper sign continuous bifunction such that:

(a) for each $x \in K, \quad F(x, x)=0$,

(b) for each $y \in K$, the set $\{x \in K: F(y, x) \in-C(y)\}$ is closed in $K$;

(c) for each $x, y, z \in K$, if $F(x, y) \in-C(x) \backslash\{0\}$ and $F(x, z) \in-C(x)$, then $F(x, u) \in-C(x) \backslash\{0\}$, for all $u \in] y, z[$,

(d) there exist a nonempty compact subset $B$ of $K$ and a nonempty convex compact subset $D$ of $K$ such that, for each $x \in K \backslash B$ there exists $y \in D$ such that $F(y, x) \notin-C(y)$.

Then, the solution set of SVEP with respect to the family $W(x)=Y \backslash(-C(x) \backslash\{0\})$, is nonempty and compact.

Proof. We define $\Gamma, \hat{\Gamma}: K \rightarrow 2^{K}$ by

$$
\begin{aligned}
& \hat{\Gamma}(y)=\{x \in K: F(x, y) \notin-C(x) \backslash\{0\}\}, \\
& \Gamma(y)=\{x \in K: F(y, x) \in-C(y)\} .
\end{aligned}
$$

By (a), $\Gamma(y)$ and $\hat{\Gamma}(y)$ are nonempty for each $y \in K$. By the $\mathcal{C}$-pseudomonotoncity of $F$ we get,

$$
\hat{\Gamma}(y) \subset \Gamma(y), \quad \forall y \in K
$$

Now, we show that $\hat{\Gamma}$ is a KKM mapping. Indeed, assume that $\hat{\Gamma}$ is not a KKM mapping, then there exist $y_{1}, y_{2}, \ldots, y_{n}$ in $K$ and $z \in \operatorname{co}\left\{y_{1}, y_{2}, \ldots, y_{n}\right\}$ such that $z \notin \bigcup_{i=1}^{n} \hat{\Gamma}\left(y_{i}\right)$. Hence, we have 


$$
F\left(z, y_{i}\right) \in-C(z) \backslash\{0\}, \quad \forall i=1,2, \ldots, n .
$$

Now, it follows from (c) that $F(z, z) \in-C(z) \backslash\{0\}$, which contradicts (a). Thus $\hat{\Gamma}$ is a KKM mapping. Now since for each $y \in K, \hat{\Gamma}(y) \subseteq \Gamma(y)$ we deduce (use the same reasoning as above with in this case if $z \notin \cup_{i=1}^{n} \Gamma\left(y_{i}\right)$ then $z \notin \Gamma\left(y_{i}\right)$ so $z \notin \hat{\Gamma}\left(y_{i}\right)$ for each $\left.1 \leq i \leq n\right)$ that $\Gamma$ is a KKM mapping. The other conditions of Theorem 1.2 are fulfilled by (b) and (d) and hence,

$$
\bigcap_{x \in K} \Gamma(x) \neq \emptyset
$$

Also, Lemma 2.13 implies that

$$
\bigcap_{x \in K} \Gamma(x)=\bigcap_{x \in K} \hat{\Gamma}(x)
$$

From (2.9) and (2.10), SVEP has a solution. Since the solution set of SVEP is $\bigcap_{x \in K} \hat{\Gamma}(x)=$ $\bigcap_{x \in K} \Gamma(x) \neq \emptyset$, then is is closed and a subset of the compact set $B$. This completes the proof.

The next corollary generalize Theorem 2.3 in [7].

Corollary 2.16. Let $K$ be a nonempty pointed closed convex subset of $X$ and $\mathcal{C}=\{C(x): x \in K\}$ is a family of proper pointed closed convex cone subsets of $Y$. Let $T: K \rightarrow L(X, Y)$ be a $\mathcal{C}$ pseudomonotone and $\mathcal{C}$-upper sign continuous bifunction such that there exist a nonempty compact subset $B$ of $K$ and a nonempty convex compact subset $D$ of $K$ such that for each $x \in K \backslash B$ there exists $y \in D$ such that $\langle T y, y-x\rangle \notin C(y)$. Then there exists an $\bar{x} \in K$ such that

$$
\langle T \bar{x}, y-\bar{x}\rangle \notin-C(\bar{x} \backslash\{0\}), \quad \forall y \in K .
$$

Proof. Let $F(x, y)=\langle T(x), y-x\rangle$. We show that $F$ satisfies the conditions of Theorem 2.15. Note (a) obviously holds. For (b) notice for each $y \in K$, the set $\{x \in K: F(y, x) \in-C(y)\}=\{x \in$ $K:\langle T(y), x-y\rangle \in-C(y)\}$ is closed in $K$, since $C(y)$ is a closed set and $T(y) \in L(X, Y)$ for each $y \in K$. To show (c) let $x, y, z \in K$ and $t \in] 0,1[$. If $F(x, y)=\langle T(x), y-x\rangle \in-C(x) \backslash\{0\}$ and $F(x, z)=\langle T(x), z-x\rangle \in-C(x)$, then

$$
\begin{gathered}
\langle T(x), t y+(1-t) z-x\rangle=\langle T(x), t(y-x)+(1-t)(z-x)\rangle= \\
t\langle T(x), y-x\rangle+(1-t)\langle T(x), z-x\rangle \in \\
-t C(x) \backslash\{0\}+(1-t)(-C(x)) \subseteq-C(x) \backslash\{0\}, \quad \text { for all } t \in] 0,1[
\end{gathered}
$$

since $-C(x) \backslash\{0\}$ is convex, note $C(x)$ is a pointed convex cone. To show (d) note for all $x \in K \backslash B$ there exists $y \in D$ such that $\langle T(y), y-x\rangle \notin C(y)$ which implies $F(y, x) \notin-C(y)$. Now apply Theorem 2.15 so there exists an $\bar{x} \in K$ such that $F(\bar{x}, y)=\langle T \bar{x}, y-\bar{x}) \notin-C(\bar{x})$ for all $y \in K$.

Received: January, 2009 . Revised: March, 2009. 


\section{References}

[1] Ansari, Q.H., Vector equilibrium problems and vector variational inequalities, In: F. Giannessi(ed) Vector variational inequalities and vector equilibria, Mathematical theories, Kluwer, Dordrecht, (2000), 1-16

[2] Bianchi, M. And Pini, R., Coercivity conditions for equilibrium problems, J. Optim. Theory Appl., 124 (2005), 79-92.

[3] Chen, G.Y. and Hou, S.H., Existence of solutions for vector variational inequalities, in: F.Giannessi(Ed), Vector variational inequalities and vector equilibria, kluwer publishers, Dordrecht, Holland, (2000), 73-86.

[4] Fakhar, M. and Zafarani, J., Generalized vector equilibrium problems for pseudomonotone multivalued bifunctions, J. Optim. Theory Appl., 126 (2005), 109-124.

[5] Fan, K., Some properties of convex sets related to fixed point theorems, Math. Ann., 266 (1984), 519-537.

[6] Fang, Y.P. And Huang, N.J., On the strong vector variational inequalities, Research Report, Department of Mathematics, Sichuan University, 2002.

[7] Fang, Y.P. And Huang, N.J., Strong vector variational inequalities in Banach spaces, Appl. Math. Lett., 19 (2006), 362-368.

[8] Giannessi, F., Vector variational inequalities and vector equilibria, Mathematical theories, Kluwer, Dordrecht, 2000.

[9] Giannessi, F., Theorems of alternative, quadratic programs, and complementarity problems, in: R. W. Cottle, Giann, J.L. Lions (Eds), Variational inequality and Complementarity problems, John Wiley and Sons, New York, (1980), 151-186.

[10] Iusem, A.N. And Sosa, W., New existence results for equilibrium problems, Nonlinear Anal., 52 (2003), 621-635.

[11] Oettli, W. and Schlager, D., Existence of equilibria for monotone multivalued mappings, Math. Meth. Oper. Res., 48 (1998), 219-228.

[12] PARK, S., Fixed points, intersections theorem, variational inequalities, and equilibrium theorems, Inter. J. Math. Math. Sci., 2 (2000), 73-93.

[13] Yang, F., Wu, C. and He, Q., Applications of Ky Fan's inequality on $\sigma$-compact set to variational inclusion and $n$ - person game theory, J. Math. Anal. Appl., 319 (2006), 177-186. 